\title{
THERAPY OF ENDOCRINE DISEASE
}

\section{Effects of chronic use of phosphodiesterase inhibitors on endothelial markers in type 2 diabetes mellitus: a meta-analysis}

\author{
Daniele Santi ${ }^{1,2}$, Elisa Giannetta ${ }^{3}$, Andrea M Isidori ${ }^{3}$, Cristiana Vitale ${ }^{4}$, \\ Antonio Aversa ${ }^{3}$ and Manuela Simoni ${ }^{1,2}$ \\ ${ }^{1}$ Unit of Endocrinology, Department of Biomedical Metabolic and Neural Sciences. University of Modena and \\ Reggio Emilia, Italy, ${ }^{2}$ Azienda USL of Modena, Italy, ${ }^{3}$ Sapienza University of Rome, Department of Experimental \\ Medicine and ${ }^{4}$ Department of Medical Sciences, IRCSS San Raffaele, Rome, Italy
}

Correspondence should be addressed to M Simoni Email

manuela.simoni@unimore.it

\begin{abstract}
Objective: Diabetes mellitus (DM) is associated with endothelial dysfunction, reducing nitric oxide-dependent vasodilation, and increasing production of pro-inflammatory factors, leading to an increased risk of long-term cardiovascular disease. As the effects of phosphodiesterase 5 inhibitors (PDE5i) on endothelial function have not been systematically investigated, we conducted a meta-analysis of available randomized clinical trials (RCTs).

Design: A thorough search of the literature was carried out. Relevant studies were considered according to RCT study design, enrollment of men with type 2 DM, chronic administration of PDE5i, and evaluation of endothelial function through both hemodynamic and endothelial inflammation-related parameters.

Results: Fifteen studies fulfilled the eligibility criteria but only six RCTs met the inclusion criteria and were analyzed for 476 diabetic men, 239 randomized to Sildenafil, and 237 to placebo respectively. Four RCTs evaluated flow-mediated dilation (FMD), demonstrating a weighted mean increase of $2.19 \%$ (95\% Cl 0.48 to 3.90). This result showed a high heterogeneity $\left(I^{2}: 98 \%\right)$. Thus, a further sub-group meta-analysis was performed and this analysis confirmed a significant, Sildenafil-related FMD improvement. Sildenafil improved endothelin 1 and high sensitivity C-reactive protein by $\sim-0.94 \mathrm{pg} / \mathrm{ml}$ and $-0.36 \mathrm{mg} / \mathrm{l}$, respectively, not reaching statistical significance $(P=0.69$ and $P=0.22$ respectively). Finally, Sildenafil administration significantly reduced serum levels of interleukin 6 (IL6, $-0.82 \mathrm{pg} / \mathrm{ml} ; 95 \% \mathrm{Cl}-1.58$ to -0.07 ). Conclusion: This meta-analysis suggests a beneficial effect of chronic PDE5i administration on endothelial function. Chronic Sildenafil administration seems to improve hemodynamic (FMD) and serum pro-inflammatory makers (IL6) in diabetic men. Larger studies are needed to confirm the effects of chronic PDE5i on endothelial function.
\end{abstract}

\section{Background}

Diabetes mellitus (DM) is one of the most common chronic diseases, with increasing incidence and an estimated prevalence of 285 million people affected worldwide (1). Type 2 DM (T2DM) had a prevalence of $2.8 \%$ in 2000 , with a projection of $4.4 \%$ in 2030 because of population growth, aging, and increased prevalence of obesity and physical inactivity (2). T2DM is characterized by a disturbance in the intermediate metabolism of carbohydrates, proteins, and lipids caused by poor insulin secretion/action, leading to chronically sustained hyperglycemia. This latter condition, together with the development of insulin resistance, promotes atherogenesis through cell proliferation at the vascular wall, (c) 2015 European Society of Endocrinology Printed in Great Britain
Published by Bioscientifica Ltd. 
producing endothelial damage (3). Thus, T2DM sustains long-term cardiovascular risk, similar to that observed among non-diabetic patients with previous myocardial infarction (4). In essence, diabetic patients have early development of abnormal endothelial function, aggressive atherosclerosis, and adverse arterial remodeling (4).

A complete understanding of nitric oxide (NO) physiology is fundamental to discover the pathogenesis of endothelial dysfunction. Diabetic endothelial dysfunction is the result of imbalance between reactive oxygen species (ROS), increased via antioxidant and glycation pathways, and the production and availability of $\mathrm{NO}$, that is reduced $(5,6,7,8)$. In addition, an increase in serum levels of vasoconstrictors, such as endothelin 1 (ET1) (9), and an impairment of several hemodynamic parameters, such as flow-mediated dilation (FMD) at brachial artery, intima-media thickness, and blood pressure (10), were described.

Erectile dysfunction (ED) seems to represent a prospective symptom of endothelial dysfunction and a consequent future vascular damage, interestingly 35-75\% of T2DM male patients $(11,12,13,14)$. The reduction in hemodynamic parameters is a common condition between patients with T2DM and ED (15).

The most commonly used treatment for ED is based on inhibitors of the enzyme phosphodiesterase 5 (PDE5): Sildenafil citrate, Tadalafil, Vardenafil cloridrate, and Avanafil. Sildenafil is the first marketed PDE5 inhibitor (PDE5i) and is extensively studied in the literature. These drugs, on-demand oral therapies to be taken before the sexual activity (16), have been deeply evaluated in clinical trials, are efficacious, and well tolerated. They facilitate erection by increasing NO availability through inhibition of cyclic guanylyl mono-phosphate (cGMP) breakdown in the endothelial cells of the penile corpus cavernous (17). However, their action is not limited to the penile tissue, so that systemic effects can be hypothesized.

It has been suggested that chronic administration of PDE5i may improve endothelial function (18). The possible mechanism(s) of action of PDE5i in improving endothelial function, could be: i) enhanced NO/cGMP signaling cascade, ii) inhibition of NADPH oxidase activity, and iii) reduction in superoxide formation (18). The antioxidant activity of PDE inhibitors was demonstrated in animal models, in which the use of different PDE5i leads to an enhanced effect on cellular cyclic nucleotide antioxidant defense, contributing to the protection against oxidative stress induced by diabetes
(19). Thus, it seems that PDE5i could restore the physiological equilibrium between mediators of vasoconstriction and vasodilation, inhibiting the catabolism of cGMP and enhancing NO levels and, consequently, vasodilation (19). These effects might result in improvement of endothelial function, reducing atherogenesis and vascular complications (19).

In recent years, several reports on the possible acute and chronic effects of PDE5i in patients with T2DM in improving endothelial function were published. However, the data available in the literature present conflicting results, challenging the interpretation of the role of PDE5i on endothelial function. Some authors found an endothelial function improvement after chronic administration of PDE5i, while other authors failed to demonstrate the beneficial effects of these drugs on endothelial parameters. Thus, to assess comprehensively the relationship between PDE5i and endothelial function in T2DM, we performed a meta-analysis of the available randomized clinical trials (RCTs), aiming at answering the following question: 'Does chronic administration of PDE5i produce endothelial function improvement in diabetic patients?'

\section{Subjects and methods}

This study was carried out according to the Cochrane Collaboration and PRISMA statement $(20,21)$.

\section{Data sources and searches}

We conducted a comprehensive literature search for English-language articles in MEDLINE (PubMed), EMBASE, Cochrane Library, SCOPUS, UpToDate, and www.clinicaltrials.gov. Search key words were: phosphodiesterase-5 inhibitors, PDE5 inhibitors, PDE-5 inhibitors, PDE-5i, PDE5i, diabetes mellitus, diabetes, DM, type 2 diabetes mellitus, type $2 \mathrm{DM}$, endothelial function, endothelial dysfunction, diabetic complications, and the Boolean functions AND and OR.

\section{Study selection}

Eligibility criteria for study selection were: i) RCT study design; ii) enrollment of men with T2DM; iii) chronic administration of any of the known PDE5i, used at their therapeutic dosage; and iv) evaluation of endothelial function. The assessment of the literature searching process is shown in Fig. 1. 


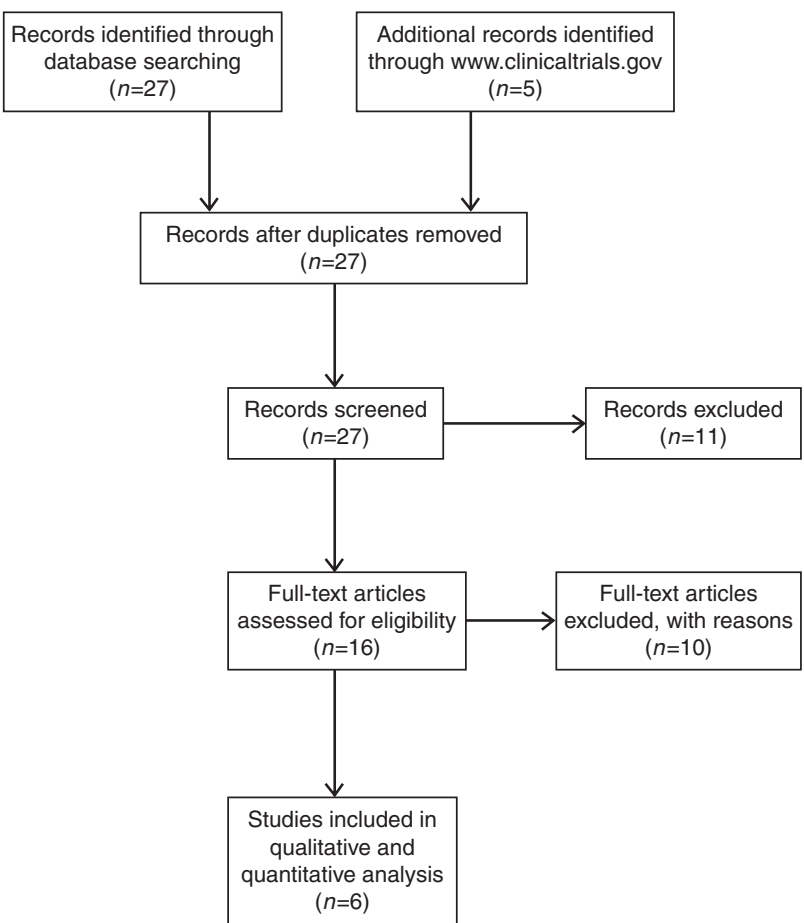

\section{Figure 1}

Study flow chart showing the search results for the studies included in the meta-analysis.

\section{Data collection process and quality}

One author (D S) extracted data from all RCTs selected with regard to study design, the year of publication, the number of included/excluded subjects, the number of dropped-out patients, and the use of intention to treat/per protocol analysis. Furthermore, the author extracted study subjects' demographics and underlying diseases, with particular attention to the time (years) elapsed from the diagnosis of T2DM. Only RCTs involving men with T2DM were considered eligible for the meta-analysis and only RCTs evaluating endothelial function, either with hemodynamic or biochemical parameters or both, were included in the meta-analytic process. Eligible RCTs should be based on a chronic administration of PDE5i. The quality of RCTs was assessed using the parameters proposed by Jadad et al. (22) and Table 1 summarizes the features of selected studies.

Several measurements of hemodynamic effects have been proposed in the literature in order to evaluate endothelial function: peak systolic velocity (23), blood flow and erythrocyte velocity $(24,25)$, arterial stiffness $(26,27,28)$, and FMD (10). The most used parameter of PDE5i efficacy on endothelial function is the improvement of the FMD score. FMD is evaluated at the brachial artery, where the diameter is measured using duplex ultrasound at baseline and after 5 min of arterial occlusion using a blood pressure cuff (29). During occlusion, the distal tissues and endothelium become ischemic, stimulating local vasodilation, which, in turn, increases blood flow in the more proximal artery during reperfusion (29). The normal response to the reactive hyperemia is a slight vasodilation of $\sim 7-15 \%$ (29). In case of endothelial dysfunction, such as in DM, the response to reactive hyperemia is blunted or even vasoconstrictive. In addition to the hemodynamic effects, PDE5i's action on endothelial cells could be evaluated by the serum biomarkers listed above. ET1 is one of the most important biochemical markers of endothelial dysfunction and it is a potent vasoconstrictor that becomes elevated in case of endothelial dysfunction, e.g. in diabetic patients (30). In addition, the $\mathrm{NO} / \mathrm{ROS}$ imbalance causes an increase in serum markers of inflammation, such as cytokines and chemocytokines, e.g. interleukins (ILs) and highsensitivity C-reactive protein (hCRP). The elevation of these markers could represent a further indication of sub-inflammation and could identify an early vascular damage. Furthermore, several studies evaluated the sexual function of diabetic patients, generally using the international index of erectile function (31). However, also in this area, no agreement exists on the questionnaire to use, as other studies report the sexual encounter profile (32) or the structured interview of erectile dysfunction (SIEDY) (33).

The primary outcomes of RCTs considered for this meta-analysis were FMD scores and ET1 serum levels. Secondary outcomes were other measurements of endothelial dysfunction, such as: IL6, IL8, hCRP, homocysteine, microalbuminuria, HbA1c, Von Willebrand factor, plasminogen activator 1 , intercellular adhesion molecule 1 (ICAM1), vascular cell adhesion molecule 1 (VCAM1), P-selectin, transforming growth factor beta (TGF $\beta$ ), monocyte chemotactic protein 1 (MCP1), and vascular endothelial growth factor (VEGF).

The second investigator (M Simoni) performed quality control checks on extracted data. The investigators, using Cochrane risk-of-bias algorithm, independently assessed the risk of bias for all trials (34). The following quality criteria and methodological details were evaluated for each trial included in the meta-analysis: i) method of randomization: only randomized studies were included in the analysis (quasi-randomized or observational trials were not included); ii) concealment of allocation: trials were considered adequate, not clear, or inadequate; iii) presence 
Table 1 Characteristics of study subjects included in the meta-analysis.

\begin{tabular}{|c|c|c|c|c|c|c|c|c|c|}
\hline References & $\begin{array}{l}\text { Number of } \\
\text { patients in } \\
\text { study group } \\
\text { (control group) }\end{array}$ & $\begin{array}{l}\text { Dropped } \\
\text { out }\end{array}$ & $\begin{array}{l}\text { Mean age } \\
\text { (years) }\end{array}$ & $\begin{array}{c}\text { Duration } \\
\text { of diabetes } \\
\text { (years) }\end{array}$ & Drugs & $\begin{array}{l}\text { Duration } \\
\text { (weeks) }\end{array}$ & $\begin{array}{c}\text { Sexual } \\
\text { parameters }\end{array}$ & $\begin{array}{l}\text { Serum } \\
\text { endothelial } \\
\text { function } \\
\text { parameters }\end{array}$ & $\begin{array}{l}\text { Endothelial } \\
\text { function } \\
\text { imaging }\end{array}$ \\
\hline (30) & $10(10)$ & 0 & $63.0 \pm 6.0$ & $6.3 \pm 3.2$ & $\begin{array}{l}\text { Sildenafil } \\
25 \mathrm{mg} \text { thrice } \\
\text { daily }\end{array}$ & 4 & IIEF5 & $\begin{array}{l}\text { IL6, VCAM1, } \\
\text { ICAM1, ET1, } \\
\text { and hCRP }\end{array}$ & $\begin{array}{r}\text { FMD and } \\
\text { RH-PAT }\end{array}$ \\
\hline (38) & $153(152)$ & 13 & - & - & $\begin{array}{l}\text { Sildenafil } \\
100 \mathrm{mg} \\
\text { daily }\end{array}$ & 4 & SEP & $\begin{array}{l}\text { IL6, IL8, and } \\
\text { CGMP }\end{array}$ & - \\
\hline (29) & $16(16)$ & 2 & $44.0 \pm 7.0$ & - & $\begin{array}{l}\text { Sildenafil } \\
25 \mathrm{mg} \text { daily }\end{array}$ & 2 & - & - & FMD \\
\hline (37) & $10(10)$ & 0 & 59.0 & 7.6 & $\begin{array}{l}\text { Sildenafil } \\
50 \text { mg daily }\end{array}$ & 10 & IIEF5 & - & FMD \\
\hline (36) & 30 (29) & 5 & $60.7 \pm 7.6$ & $6.7 \pm 5.5$ & $\begin{array}{l}\text { Sildenafil } \\
100 \mathrm{mg} \\
\text { daily }\end{array}$ & 12 & - & $\begin{array}{l}\text { ET1, MCP1, } \\
\text { TGF } \beta \text {, and } \\
\text { VEGF }\end{array}$ & FMD \\
\hline (35) & $20(20)$ & 0 & $48.3 \pm 4.52$ & - & $\begin{array}{l}\text { Sildenafil } \\
\quad 50 \text { mg daily }\end{array}$ & 4 & IIEF15 & hCRP & - \\
\hline
\end{tabular}

CGMP, cyclic guanylyl mono-phosphate; ET1, endothelin 1; FMD, flow-mediated dilation; hCRP, high C-reactive protein; HRV, heart rate variability; ICAM1, intercellular chemotactic protein 1; IIEF, international index of erectile function; IL, interleukin; IMT, intima-media thickness; MCP1, monocyte chemotactic protein 1; RH-PAT, reactive hyperemia by peripheral arterial tonometry; SEP, sexual encounter profile; TGF $\beta$, transforming growth factor beta; VCAM1, vascular cell adhesion molecule 1; VEGF, vascular endothelial growth factor.

or absence of blinding to treatment allocation; iv) duration and type of follow-up; v) the number of participants recruited, randomized, analyzed, or lost to follow-up; vi) timing of trial; vii) whether an intention to treat analysis was done; viii) whether a power calculation was done; ix) source funding; and $\mathrm{x}$ ) criteria for including a participant and assessing outcomes.

\section{Data synthesis and analysis}

The meta-analysis of the data extracted from the included studies was conducted using the Review Manager 5.2 Software (Copenaghen, Denmark) (version 5.2.4 Copenhagen: The Nordic Cochrane Centre, The Cochrane Collaboration, 2012). Data were combined using the random effect model, providing a more conservative estimate of the overall effect, especially relevant when studies were of different design and duration. Weighted mean differences and 95\% CIs were estimated for each endpoint considered. Heterogeneity between the results of different studies was examined by inspecting the scatter in the data points and the overlap in their CIs and by performing $\chi^{2}$ tests and $I^{2}$ statistics. Given the heterogeneity of the studies enclosed and considering the wide range of markers of inflammation studied, several subgroup analyses were performed using different endothelial parameters. Values of $P<0.05$ were considered statistically significant.
Meta-regression analysis was conducted using the 'Statistical Package for the Social Sciences' Software for Windows (version 16.0; SPSS, Inc.).

\section{Results}

\section{Study selection}

Figure 1 shows the literature searching process conducted from November 2013 to January 2014. We identified 32 potentially relevant studies. Of these, five studies were excluded because they are present in more than one database and 11 were excluded based on the information given in the abstract. A total of 16 trials were finally identified by the strategy research. Overall, 550 diabetic men were enrolled in the meta-analytic process. All trials were thoroughly appraised for their eligibility in the metaanalysis and their methodological quality. Six studies met the inclusion criteria $(29,30,35,36,37,38)$ (Table 1$)$. Ten studies were excluded from the final analysis as they did not fulfill inclusion criteria. One RCT was excluded from the analysis as it studied the efficacy and safety of a new PDE5i (Avanafil) in diabetic patients, but it did not analyze endothelial function (39). Two RCTs were excluded as they provided an evaluation of endothelial function after acute rather than chronic treatment with a PDE5i and because of quasi-randomization $(40,41)$. One RCT was 
excluded because it investigated the synergic effect of propionyl L-carnitine (PLC) plus Sildenafil in reducing monocyte oxidative activity and endothelial dysfunction markers in diabetic patients with ED (42). Thus, in this latter study, there was a confounding factor, i.e. the possible protective effect of PLC on endothelial function (42). Another clinical trial employing Tadalafil was excluded as it had a retrospective design and was not placebo controlled (43). An RCT considering PDE5i and T2DM was excluded as only seminal vesicle volumes were evaluated and parameters of endothelial health were not considered (44). Two studies enrolled only patients with $\mathrm{ED}$, with or without T2DM and presented an open-label study design $(45,46)$. One trial was conducted enrolling naïve T2DM patients with ED treated with daily Tadalafil vs placebo (47). We excluded this trial because it was not possible to extract the data concerning the only 25 diabetic patients out of a total number of 215 subjects enrolled (47). Furthermore, this study evaluated the effect of Tadalafil once daily used at a dosage lower than the therapeutic dosage employed in the other RCTs included (47). Finally, the last RCT excluded from the meta-analysis did not randomize patients and primarily evaluated NO metabolites in penile corpora cavernosa (48).

\section{Study characteristics}

All trials included in the meta-analysis $(29,30,35,36$, 37,38 ) enrolled men with T2DM based on the definition of WHO (49). T2DM was defined in the same way in the six included studies, but a different duration of the disease was reported in the inclusion criteria of each RCT (Table 1). The different duration of T2DM in each trial, together with the differences among inclusion/exclusion criteria used, could represent the most important source of heterogeneity. These are typical selection bias in studies involving diabetic patients, considering the natural evolution of the disease.

Selected trials gave details as follows: $\sim 472$ diabetic men, 237 randomized to PDE5i and 235 to placebo. Only one study included had a cross-over design (29).

All trials included in the meta-analysis $(29,30,35,36$, 37,38 ) considered the chronic administration of PDE5i. In particular, all studies provided the use of the same PDE5i: Sildenafil citrate. However, different study designs were applied and the duration of treatment lasted from 2 (29) to 12 weeks (36). This difference in the treatment duration could represent another important source of heterogeneity. Furthermore, the study design of most trials included did not provide an evaluation after follow-up. Only one RCT evaluated the effects during treatment and after adequate follow-up after withdrawal of Sildenafil (30). These characteristics resulted in incomplete information about the follow-up of these patients and might prevent recognition of long-term effect of PDE5i on endothelial function.

The outcomes of the RCTs considered in this metaanalysis were divided into two different classes: hemodynamic parameters and serum biochemical markers. The first group included the measurement of FMD, reported in four RCTs $(29,30,36,37)$, and reactive hyperemia by peripheral arterial tonometry, reported in one RCT (30). The second group of endpoints comprised ET1, IL6, and hCRP, reported in at least three studies $(17,30,36)$, as well as cGMP, IL8, ICAM1, VCAM1, MCP1, TGF $\beta$, and VEGF, reported in one RCT.

\section{Risk of bias in included studies}

Allocation (selection bias) $>$ Two trials stated precisely the methodology of randomization used $(35,36)$. Four trials did not specify the method of randomization, even if they stated that patients were randomly assigned to the study or control group.

Blinding (performance bias and detection bias) $>$ All six RCTs included in the meta-analysis were double blinded.

Incomplete outcome data (attrition bias) $\downarrow$ Three RCTs had dropped out after randomization, but none reported whether intention to treat or per protocol analysis was performed $(29,36,38)$.

Selective reporting (reporting bias) $\downarrow$ The primary endpoints described in the aims of each RCT were reported in the result section of each trial. Several studies included secondary endpoints, which, however, were not always reported.

Other potential sources of bias $>$ Power analysis was made only in two RCTs included in the meta-analysis $(35,36)$. Other RCTs considered in the meta-analysis did not report a calculation of the sample size in the study design.

\section{Efficacy data: PDE5i vs placebo}

Considering the wide variability of endpoints evaluated in these studies, it was not possible to conduct a single comprehensive analysis. Thus, four subgroup meta-analyses were performed. The first considered four RCTs evaluating FMD in 62 patients and 56 controls $(29,30,36,37)$ and 
showed significantly improved FMD values after Sildenafil treatment. The mean improvement was $2.19 \%$ with CI 0.48 to $3.90(P<0.001$; Fig. 2$) . \chi^{2}$ was 166.40 and $I^{2}$ statistics was $98 \%$. The $I^{2}$ statistics answers the question: 'What proportions of the observed variance reflect real differences in effect size?' It is a measure of inconsistency across the findings of the studies, and it is not a measure of the real variation across the underlying true effects. Thus, the $I^{2}$ statistics showed a certain degree of inconsistency across this result. In order to evaluate this heterogeneity, we analyzed each single RCT, especially considering the inclusion criteria. Three of the four RCTs evaluated in this subgroup considered men with a history of long-term DM and with HbA1c over the upper range of normality, suggesting a possible effect of DM on endothelial function at baseline $(29,36,37)$. On the other hand, Aversa et al. (30) enrolled men with ED and DM in good metabolic compensation, with HbA1c serum levels around the normal range (6.4\%). Considering this variability in patient selection, we excluded the latter study (30) and performed a new sub-group meta-analysis (Fig. 3). The forest plot confirmed the significant improvement in FMD scores in the study group $(0.34, \mathrm{CI} 0.27$ to $0.42, P<0.001) \cdot \chi^{2}$ was 1.81 and $I^{2}$ statistics was $0 \%$.

Given the wide range of markers used to evaluate endothelial health in the RCTs included, we were unable to perform one comprehensive meta-analysis. Thus, we tried to subdivide the overall effect of PDE5i on endothelial health, considering each endothelial marker analyzed in at least two studies. We performed a subgroup meta-analysis considering the two studies in which ET1 was evaluated, including 40 patients and 39 controls $(30,36)$, showing no effect of PDE5i on this parameter. The mean difference was $-0.94 \mathrm{pg} / \mathrm{ml}, \mathrm{CI}-5.49$ to 3.62 $\left(P=0.690\right.$; Fig. 4). $\chi^{2}$ was 1.01 and $I^{2}$ statistics was $1 \%$.
The third sub-group meta-analysis considered two RCTs evaluating hCRP in 30 patients and 30 controls $(30,35)$. The PDE5i use did not result in any significant reduction in hCRP plasma levels (mean difference $-0.36 \mathrm{mg} / \mathrm{l}$, CI from -0.91 to $0.19, P=0.220$; Fig. 5).

Finally, the last meta-analysis involved two studies including 163 patients and 162 controls analyzing IL6 serum levels $(30,38)$. This analysis showed that the use of PDE5i reduced IL6 serum levels significantly more than placebo (mean difference $-0.82 \mathrm{pg} / \mathrm{ml}$, CI from -1.58 to -0.07 , $P<0.001$; Fig. 6). $\chi^{2}$ was 28.53 and $I^{2}$ statistics was $96 \%$.

A logistic multivariate meta-regression was conducted comparing the variation of FMD and ET1. No significant correlation was found between these two parameters considering the weight of the studies enrolled $(P=0.997)$. Furthermore, a meta-regression was conducted considering the variation of FMD and hCRP. No significant correlation was found $(P=0.986)$.

\section{Discussion}

To the best of our knowledge, no previous meta-analysis focused on the effects of PDE5i on endothelial dysfunction in T2DM. Herein, for the first time, we report the results of a meta-analysis evaluating differences in endothelial function parameters in diabetic men after chronic treatment with PDE5i or placebo. The diversity of endpoints considered does not permit a comprehensive metaanalytic approach to this problem. However, we could evaluate a large number of diabetic patients chronically treated with PDE5i and decided to perform subgroup meta-analysis, considering only parameters analyzed in more than one RCT.

First, we showed an improvement of FMD scores after chronic treatment with Sildenafil in 162 diabetic patients,

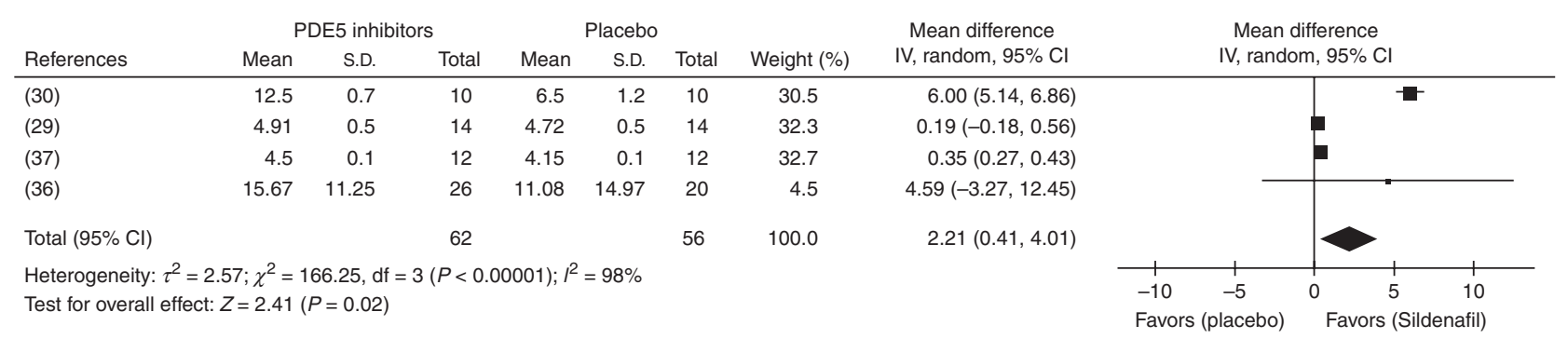

\section{Figure 2}

Forest plot including the result of the subgroup meta-analysis, comparing the effects of PDE5 inhibitors over placebo in T2DM patients. FMD values, expressed in \%, were considered.

Diamond indicates the overall summary estimate for the analysis (width of the diamond represents the $95 \% \mathrm{Cl}$ ) and boxes the weight of individual studies in the pooled analysis. 


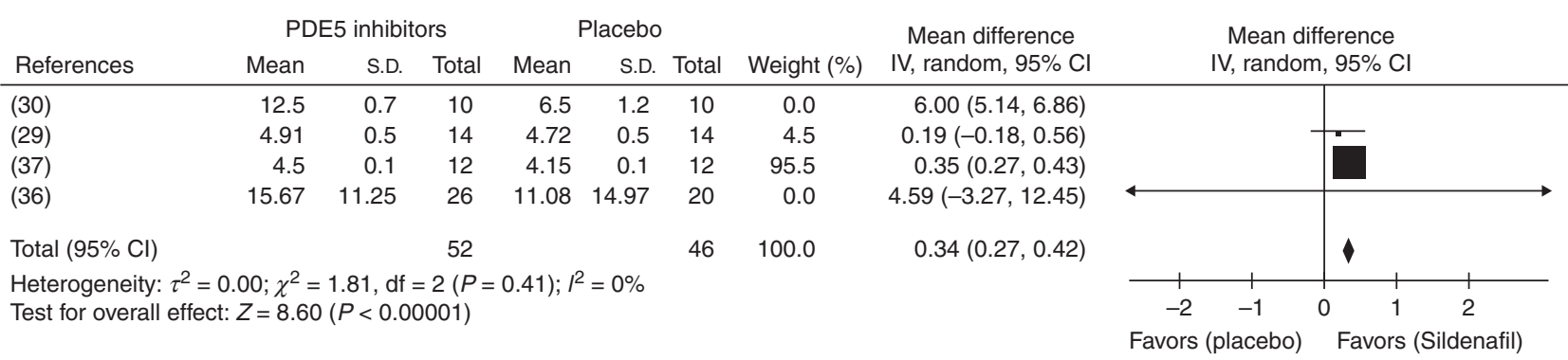

\section{Figure 3}

Forest plot including the result of the subgroup meta-analysis, comparing the effects of PDE5 inhibitors over placebo in T2DM patients, evaluating only three studies after the evaluation of heterogeneity. FMD values, expressed in \%, were considered.

independent of the treatment length $(P<0.001$; Fig. 2). However, the heterogeneity found in this analysis represents an important drawback in drawing conclusions. The possible explanation of this variability between studies derives from the natural history of DM. In fact, this is a chronic disease that leads to an endothelial damage/dysfunction depending on the duration of the disease itself. Thus, patients with long-lasting DM are expected to have a worse endothelial function than patients with newly diagnosed DM. Furthermore, the 'ceiling' effect of PDE5i may play some roles (47). In other words, the beneficial effect on endothelial health of PDE5i is higher when patients have an advanced damage of endothelium due to long-term DM (50). The residual meta-analysis, excluding one RCT, confirmed the beneficial effect of Sildenafil on FMD (Fig. 3). We conclude that chronic Sildenafil treatment improves hemodynamic parameters in DM. This result seems to be in contrast with previous studies demonstrating that PDE5i are not effective in improving endothelial function in a subset of
Diamond indicates the overall summary estimate for the analysis (width of the diamond represents the $95 \% \mathrm{Cl}$ ) and boxes the weight of individual studies in the pooled analysis.

healthy subjects (50). Therefore, we do not know whether PDE5i could have a protective role against the development of diabetic endothelial dysfunction. Some studies of the literature support the hypothesis that PDE5i have a beneficial effect on hemodynamic parameters. For example, one-time administration of PDE5i increases FMD in patients with DM (41), ED (15), congestive heart failure (51), and in habitual cigaret smokers $(52,53)$. However, such trials are not comparable for the enrollment of patients, with a wide variability of inclusion criteria. Furthermore, no studies evaluated the efficacy of PDE5i after an adequate follow-up after drug withdrawal. Thus, it remains unknown whether this beneficial effect of PDE5 $i$ is maintained over time and for how long. A recent metaanalysis involving a mixed population of 1622 patients has shown that long-term daily PDE5i administration improves several cardiac outcomes including ejection fraction, cardiac output and diastolic function (54).

The resulting serum levels of IL6 became significantly reduced after chronic Sildenafil treatment (Fig. 6). IL6

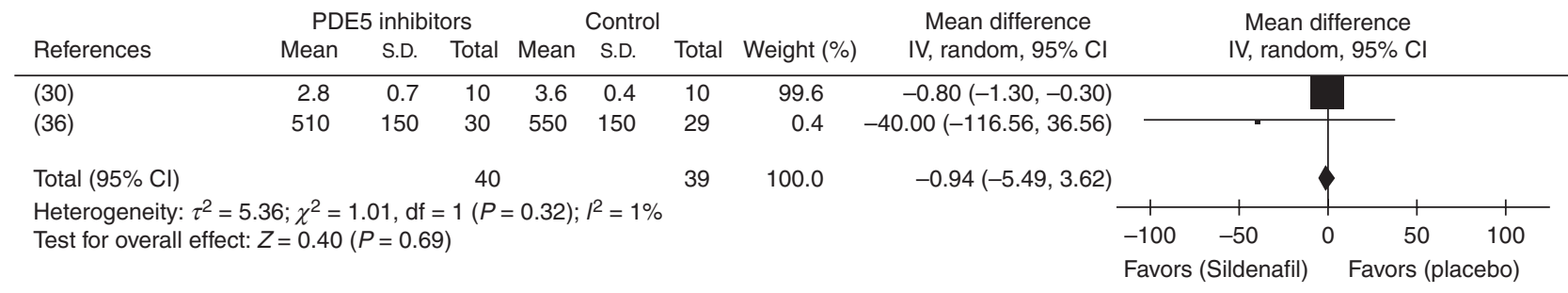

\section{Figure 4}

Forest plot including the result of the subgroup meta-analysis, comparing the effects of PDE5 inhibitors over placebo in T2DM patients. ET1 serum levels, expressed in $\mathrm{pg} / \mathrm{ml}$, were considered.
Diamond indicates the overall summary estimate for the analysis (width of the diamond represents the $95 \% \mathrm{Cl}$ ) and boxes the weight of individual studies in the pooled analysis. 


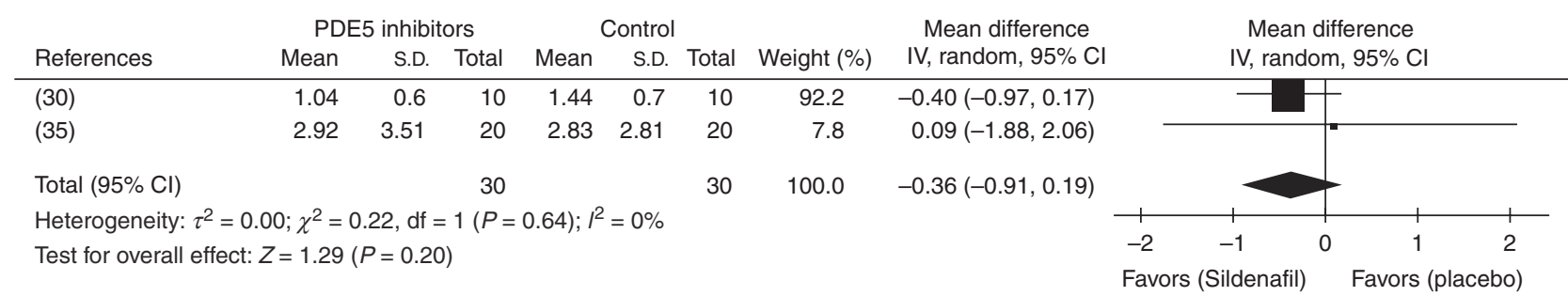

\section{Figure 5}

Forest plot including the result of the subgroup meta-analysis, comparing the effects of PDE5 inhibitors over placebo in T2DM patients. hCRP plasma levels, expressed in $\mathrm{mg} / \mathrm{l}$, were considered. Diamond indicates the overall summary estimate

belongs to the wide family of cytokines involved in the development of a pro-inflammatory state resulting in vascular inflammation and atherosclerosis (55). Furthermore, IL6 has been recently proposed as a regulator of cell senescence (56). Our results suggest that chronically administered PDE5i could lead to a reduction in serum levels of pro-inflammatory cytokine. The reduction in IL6 serum levels could retard the development of a proinflammatory state, possibly preventing atherosclerosis. However, meta-regression failed to find any correlation between the variation in FMD scores and IL6 levels, confirming the difficulty of relating this serum biochemical marker to hemodynamic parameters in T2DM patients. A larger study analyzing several pro-inflammatory cytokines in depth could be helpful to elucidate whether PDE5i exert anti-inflammatory effects.

Considering the two studies evaluating ET1 serum levels as a marker of endothelial dysfunction $(30,36)$, the meta-analytic result of a total of 99 patients showed a reduction in ET1 serum levels, which, however, was not statistically significant $(P=0.10$; Fig. 4$)$. This result is for the analysis (width of the diamond represents the $95 \% \mathrm{Cl}$ ) and boxes the weight of individual studies in the pooled analysis.

probably related to the difficulties in assessing accurately ET1 by the available assays. In this setting, the two trials considered used different laboratory assays. Giannetta et al. (36) used a quantitative sandwich enzyme immunoassay (Quantikine, R\&D Systems, Abingdon, UK), finding mean values of the order of $0.5 \mathrm{ng} / \mathrm{ml}$. Conversely, Aversa et al. (30) measured ET1 levels by RIA, after extraction through absorption column cartridge (Sep-pack C18; Waters, MA, USA), obtaining values of the order of $3 \mathrm{pg} / \mathrm{ml}$. In the literature, a wide range of ET1 assays with different sensibilities and specificities exist and the results obtained with these methodologies are difficult to reproduce in different experimental settings. Thus, the methodology chosen for ET1 measurement is of utmost importance, considering that, in dependence thereof, the results can vary of at least one order of magnitude. This difference could justify the lack of significant variations of ET1 levels in the meta-analyzed RCT. The meta-regression conducted considering FMD and ET1 revealed no correlations between these two markers of endothelial function, suggesting again that this biochemical serum marker

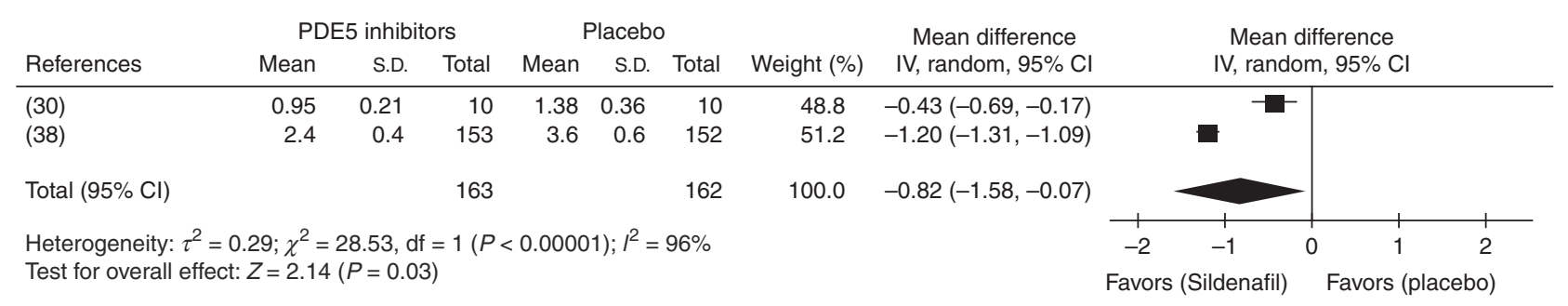

\section{Figure 6}

Forest plot including the result of the subgroup meta-analysis, comparing the effects of PDE5 inhibitors over placebo in T2DM patients. IL6 serum levels, expressed in $\mathrm{pg} / \mathrm{ml}$, were considered.
Diamond indicates the overall summary estimate for the analysis (width of the diamond represents the $95 \% \mathrm{Cl}$ ) and boxes the weight of individual studies in the pooled analysis. 
and the hemodynamic parameters might not be related. Two studies evaluated hCRP $(30,36)$. hCRP is a known marker of inflammation, but doubts remain on its validity as an endothelial function parameter in diabetic patients. In particular, Grover-Paez et al. (35) speculated that the absence of significant variations in hCRP serum levels in the study group could be due to the short duration of the study. By contrast, Aversa et al. (30) found a significant reduction of $\sim 20 \%$ in serum levels of hCRP in the study group. The present meta-analysis based on 60 patients found a non-significant reduction in hCRP levels in the study group (Fig. 5). Thus, after chronic treatment with PDE5i, significant decreases were found neither for ET1 nor for hCRP. This lack of significance is probably due to the difficulty to measure these markers in serum, together with the differences in patients enrolled in the two studies considered in each sub-group meta-analysis. Furthermore, this result derives from only two studies, limiting the strength of this estimation.

One trial included in our meta-analysis evaluated many serum biochemical markers. This study considered 306 diabetic patients, demonstrating that the chronic treatment with Sildenafil for 12 weeks improves endothelial function in the cardiovascular system beyond the sexual sphere (38). The primary endpoints of this study were three serum markers of endothelial function: cGMP, IL6, and IL8 (38). However, only cGMP serum levels improved significantly after 4 weeks of Sildenafil treatment, whereas IL6 and IL8 did not (38). This result on IL6 is in contrast with the meta-analytical finding, probably due to the methodological limitations of the cytokine assays, inaccurate in clinical studies and, generally, with low sensitivity. Conversely, Aversa et al. (30) demonstrated a significant reduction in VCAM1 and ICAM1 in 20 diabetic men after 3 weeks of Sildenafil treatment. VCAM1 and ICAM1 are inflammatory target proteins and their levels are related to the inflammatory state $(57,58)$. However, further studies are needed to confirm the cardiac effects of PDE5i.

The importance of finding a drug able to prevent the endothelial dysfunction in T2DM is due to its known role in the atherogenetic process (59). In fact, endothelial dysfunction plays a key role in the pathogenesis of atherosclerosis and of micro- and macro-vascular complications typical of DM (60). At the same time, endothelial dysfunction is the commonest cause of vasculogenic ED (61). ED is typical of T2DM and may be considered as a sentinel symptom of occult cardiovascular disease (62). For these reasons, finding a drug possibly able to reduce endothelial dysfunction could prevent numerous complications and adverse outcomes in diabetic patients. Several mechanisms could be altered in endothelial dysfunction, but the primary alteration is a reduction in NO levels (63). Thus far, little attention was given to mechanisms through which PDE5i might benefit endothelial function beyond the NO-cGMP pathway, e.g. alteration of serum biomarkers, increased levels of endothelial progenitor cells, ischemia-reperfusion protection mechanisms, and other actions specific to diabetic patients (64). The results of our meta-analysis overall confirm the possible positive role of PDE5i on endothelial function, as measured by FMD. However, they also indicate that a reproducible and validated serum marker of endothelial health was not identified thus far. In fact, it seems that hemodynamic parameters are better related to endothelial health than biochemical markers, possibly because of the heterogeneity of the biochemical markers assessed. The demonstration of a beneficial effect of PDE5i against atherosclerosis in patients at risk, such as diabetic patients, could justify the evaluation of the same effect in other populations, e.g. women, or men with a high risk of atherogenesis due to causes other than diabetes. Furthermore, this meta-analysis shows, for the first time, that chronic, daily treatment with PDE5i could have a beneficial effect. Therefore, use of PDE5i may become a new therapeutic approach, so far approved for on-demand treatment of ED.

The limits of our meta-analysis are primarily due to the heterogeneity of the studies. All RCTs enrolled diabetic men, but the inclusion and exclusion criteria were different, as well as the study designs. All studies employed the same PDE5i (i.e. Sildenafil citrate), but in different doses, regimens, and duration. Furthermore, a possible attrition bias could be present in each RCT, as no information about dropped-out patients was given. Taken together, all these aspects limit the strength of the result and could mask the beneficial effect of PDE5i on endothelial health. Finally, follow-up was considered only in one study (30). Thus, we are unable to evaluate whether the inflammatory state could be restored after the withdrawal of PDE5i and when this could happen. Each study, taken alone, demonstrates that the use of PDE5i in diabetic men progressively improves endothelial function and increases the percentage of patients with a significant improvement in endothelial function. However, it should be recognized that a different duration of T2DM results in different comorbidity prevalence. This could represent a confounding factor, which cannot be accounted for by the meta-analyzed data, even if it could be considered a relevant covariate. 


\section{Conclusions}

PDE5i are approved in ED and pulmonary hypertension and their use is under investigation for further diseases, such as asthma, acute ischemic stroke, cancer, systemic inflammation, claudicatio intermittens, and chronic obstructive pulmonary disease. The results of the RCTs available in the literature and of this meta-analysis indicate a possible influence of PDE5i on the modification of the natural course of DM. In fact, we show that chronic treatment with Sildenafil reduces the FMD scores and IL6 serum levels, but a definitive demonstration of the therapeutic role of PDE5i in the prevention of endothelial dysfunction in DM is far to be elucidated.

Future RCTs should be conducted in more homogeneous population of diabetic men with the aim of identifying the best biochemical or hemodynamic parameter of endothelial function. The development of a reliable predictor of endothelial health could represent a real advantage in the management of T2DM and its comorbidities.

\section{Declaration of interest}

The authors declare that there is no conflict of interest that could be perceived as prejudicing the impartiality of the research reported.

\section{Funding}

This research did not receive any specific grant from any funding agency in the public, commercial or not-for-profit sector. D Santi is a recipient of a PhD fellowship from the Doctorate School of Clinical and Experimental Medicine at the University of Modena and Reggio Emilia.

\section{References}

1 Moreno G \& Mangione CM. Management of cardiovascular disease risk factors in older adults with type 2 diabetes mellitus: 2002-2012 literature review. Journal of the American Geriatrics Society 201361 2027-2037. (doi:10.1111/jgs.12513)

2 Wild S, Roglic G, Green A, Sicree R \& King H. Global prevalence of diabetes: estimates for the year 2000 and projections for 2030. Diabetes Care 200427 1047-1053. (doi:10.2337/diacare.27.5.1047)

3 Matthaei S, Stumvoll M, Kellerer M \& Haring HU. Pathophysiology and pharmacological treatment of insulin resistance. Endocrine Reviews 2000 21 585-618.

4 Haffner SM, Lehto S, Ronnemaa T, Pyorala K \& Laakso M. Mortality from coronary heart disease in subjects with type 2 diabetes and in nondiabetic subjects with and without prior myocardial infarction. New England Journal of Medicine 1998339 229-234. (doi:10.1056/ NEJM199807233390404)

5 Hellsten Y, Nyberg M, Jensen LG \& Mortensen SP. Vasodilator interactions in skeletal muscle blood flow regulation. Journal of Physiology 2012590 6297-6305. (doi:10.1113/jphysiol.2012.240762)

6 Wen Y, Skidmore JC, Porter-Turner MM, Rea CA, Khokher MA \& Singh BM. Relationship of glycation, antioxidant status and oxidative stress to vascular endothelial damage in diabetes. Diabetes, Obesity \& Metabolism 20024 305-308. (doi:10.1046/j.1463-1326. 2002.00212.x)

7 Hakim LS \& Goldstein I. Diabetic sexual dysfunction. Endocrinology and Metabolism Clinics of North America 199625 379-400. (doi:10.1016/ S0889-8529(05)70329-7)

8 Taylor AA. Pathophysiology of hypertension and endothelial dysfunction in patients with diabetes mellitus. Endocrinology and Metabolism Clinics of North America 200130 983-997. (doi:10.1016/S08898529(05)70223-1)

9 Ahn GJ, Yu JY, Choi SM, Kang KK, Ahn BO, Kwon JW, Kang SK, Lee BC $\&$ Hwang WS. Chronic administration of phosphodiesterase 5 inhibitor improves erectile and endothelial function in a rat model of diabetes. International Journal of Andrology 200528 260-266. (doi:10.1111/j.13652605.2005.00537.x)

10 Poredos P \& Jezovnik MK. Testing endothelial function and its clinical relevance. Journal of Atherosclerosis and Thrombosis 201320 1-8. (doi:10.5551/jat.14340)

11 Cho NH, Ahn CW, Park JY, Ahn TY, Lee HW, Park TS, Kim IJ, Pomerantz K, Park C, Kimm KC et al. Prevalence of erectile dysfunction in Korean men with type 2 diabetes mellitus. Diabetic Medicine 200623 198-203. (doi:10.1111/j.1464-5491.2005.01789.x)

12 Grover SA, Lowensteyn I, Kaouache M, Marchand S, Coupal L, DeCarolis E, Zoccoli J \& Defoy I. The prevalence of erectile dysfunction in the primary care setting: importance of risk factors for diabetes and vascular disease. Archives of Internal Medicine 2006166 213-219. (doi:10.1001/archinte.166.2.213)

13 Sun P, Cameron A, Seftel A, Shabsigh R, Niederberger C \& Guay A. Erectile dysfunction - an observable marker of diabetes mellitus? A large national epidemiological study. Journal of Urology $2006 \mathbf{1 7 6}$ 1081-1085 (discussion 1085). (doi:10.1016/j.juro.2006.04.082)

14 De Angelis L, Marfella MA, Siniscalchi M, Marino L, Nappo F, Giugliano F, De Lucia D \& Giugliano D. Erectile and endothelial dysfunction in type II diabetes: a possible link. Diabetologia 200144 1155-1160. (doi:10.1007/s001250100616)

15 Mazo E, Gamidov S \& Iremashvili V. The effect of vardenafil on endothelial function of brachial and cavernous arteries. International Journal of Impotence Research 200618 464-469. (doi:10.1038/sj.ijir. 3901454)

16 Carson CC, Burnett AL, Levine LA \& Nehra A. The efficacy of sildenafil citrate (Viagra) in clinical populations: an update. Urology $2002 \mathbf{6 0}$ 12-27. (doi:10.1016/S0090-4295(02)01687-4)

17 Goldstein I, Lue TF, Padma-Nathan H, Rosen RC, Steers WD \& Wicker PA. Oral sildenafil in the treatment of erectile dysfunction. Sildenafil Study Group. New England Journal of Medicine 1998338 1397-1404. (doi:10.1056/NEJM199805143382001)

18 Schafer A, Fraccarollo D, Pfortsch S, Flierl U, Vogt C, Pfrang J, Kobsar A Renne T, Eigenthaler M, Ertl G et al. Improvement of vascular function by acute and chronic treatment with the PDE-5 inhibitor sildenafil in experimental diabetes mellitus. British Journal of Pharmacology 2008153 886-893. (doi:10.1038/sj.bjp.0707459)

19 Milani E, Nikfar S, Khorasani R, Zamani MJ \& Abdollahi M. Reduction of diabetes-induced oxidative stress by phosphodiesterase inhibitors in rats. Comparative Biochemistry and Physiology. Toxicology \& Pharmacology 2005140 251-255. (doi:10.1016/j.cca.2005.02.010)

20 Moher D, Altman DG, Liberati A \& Tetzlaff J. PRISMA statement Epidemiology 201122128 (author reply 128). (doi:10.1097/EDE. Ob013e3181fe7825)

21 Moher D, Liberati A, Tetzlaff J \& Altman DG. Preferred reporting items for systematic reviews and meta-analyses: the PRISMA statement. International Journal of Surgery 20108 336-341. (doi:10.1016/j.ijsu. 2010.02.007)

22 Jadad AR, Moore RA, Carroll D, Jenkinson C, Reynolds DJ, Gavaghan DJ $\&$ McQuay HJ. Assessing the quality of reports of randomized clinical trials: is blinding necessary? Controlled Clinical Trials 199617 1-12. (doi:10.1016/0197-2456(95)00134-4) 
23 Sommer F, Klotz T \& Engelmann U. Improved spontaneous erectile function in men with mild-to-moderate arteriogenic erectile dysfunction treated with a nightly dose of sildenafil for one year: a randomized trial. Asian Journal of Andrology 20079 134-141. (doi:10.1111/j. 1745-7262.2007.00233.x)

24 Attina TM, Malatino LS, Maxwell SR, Padfield PL \& Webb DJ. Phosphodiesterase type 5 inhibition reverses impaired forearm exercise-induced vasodilatation in hypertensive patients. Journal of Hypertension 200826 501-507. (doi:10.1097/HJH.0b013e3282f382ff)

25 Kaufmann BA, Bernheim AM, Kiencke S, Fischler M, Sklenar J, Mairbaurl H, Maggiorini M \& Brunner-La Rocca HP. Evidence supportive of impaired myocardial blood flow reserve at high altitude in subjects developing high-altitude pulmonary edema. American Journal of Physiology. Heart and Circulatory Physiology 2008294 H1651-H1657. (doi:10.1152/ajpheart.00760.2007)

26 Hirata K, Adji A, Vlachopoulos C \& O'Rourke MF. Effect of sildenafil on cardiac performance in patients with heart failure. American Journal of Cardiology 200596 1436-1440. (doi:10.1016/j.amjcard.2005.06.091)

27 Oliver JJ, Melville VP \& Webb DJ. Effect of regular phosphodiesterase type 5 inhibition in hypertension. Hypertension $2006 \mathbf{4 8}$ 622-627. (doi:10.1161/01.HYP.0000239816.13007.c9)

28 Vlachopoulos C, Hirata K \& O'Rourke MF. Effect of sildenafil on arterial stiffness and wave reflection. Vascular Medicine 20038 243-248. (doi:10.1191/1358863x03vm509ra)

29 Desouza C, Parulkar A, Lumpkin D, Akers D \& Fonseca VA. Acute and prolonged effects of sildenafil on brachial artery flow-mediated dilatation in type 2 diabetes. Diabetes Care 200225 1336-1339. (doi:102337/diacare.25.8.1336)

30 Aversa A, Vitale C, Volterrani M, Fabbri A, Spera G, Fini M \& Rosano GM. Chronic administration of Sildenafil improves markers of endothelial function in men with type 2 diabetes. Diabetic Medicine 200825 37-44. (doi:10.1111/j.1464-5491.2007.02298.x)

31 Rosen RC, Riley A, Wagner G, Osterloh IH, Kirkpatrick J \& Mishra A. The international index of erectile function (IIEF): a multidimensional scale for assessment of erectile dysfunction. Urology 199749 822-830. (doi:10.1016/S0090-4295(97)00238-0)

32 Yang L, Qian S, Liu L, Pu C, Yuan H, Han P \& Wei Q. Phosphodiesterase-5 inhibitors could be efficacious in the treatment of erectile dysfunction after radiotherapy for prostate cancer: a systematic review and metaanalysis. Urologia Internationalis 201390 339-347. (doi:10.1159/ 000343730)

33 Petrone L, Mannucci E, Corona G, Bartolini M, Forti G, Giommi R \& Maggi M. Structured interview on erectile dysfunction (SIEDY): a new, multidimensional instrument for quantification of pathogenetic issues on erectile dysfunction. International Journal of Impotence Research 2003 15 210-220. (doi:10.1038/sj.ijir.3901006)

34 Chandler J, Clarke M \& Higgins J. The Cochrane Collaboration. Cochrane Methods. UK: John Wiley \& Sons Ltd, 2012.

35 Grover-Paez F, Villegas Rivera G \& Guillen Ortiz R. Sildenafil citrate diminishes microalbuminuria and the percentage of A1c in male patients with type 2 diabetes. Diabetes Research and Clinical Practice 2007 78 136-140. (doi:10.1016/j.diabres.2007.02.006)

36 Giannetta E, Isidori AM, Galea N, Carbone I, Mandosi E, Vizza CD, Naro F, Morano S, Fedele F \& Lenzi A. Chronic inhibition of cGMP phosphodiesterase $5 \mathrm{~A}$ improves diabetic cardiomyopathy: a randomized, controlled clinical trial using magnetic resonance imaging with myocardial tagging. Circulation 2012125 2323-2333. (doi:10.1161/ CIRCULATIONAHA.111.063412)

37 Deyoung L, Chung E, Kovac JR, Romano W \& Brock GB. Daily use of sildenafil improves endothelial function in men with type 2 diabetes. Journal of Andrology 201233 176-180. (doi:10.2164/jandrol.111.013367)

38 Burnett AL, Strong TD, Trock BJ, Jin L, Bivalacqua TJ \& Musicki B. Serum biomarker measurements of endothelial function and oxidative stress after daily dosing of sildenafil in type 2 diabetic men with erectile dysfunction. Journal of Urology 2009181 245-251. (doi:10.1016/j.juro. 2008.09.005)
39 Goldstein I, Jones LA, Belkoff LH, Karlin GS, Bowden CH, Peterson CA, Trask BA \& Day WW. Avanafil for the treatment of erectile dysfunction: a multicenter, randomized, double-blind study in men with diabetes mellitus. Mayo Clinic Proceedings 201287 843-852. (doi:10.1016/j. mayocp.2012.06.016)

40 Hatzichristou D, Gambla M, Rubio-Aurioles E, Buvat J, Brock GB, Spera G, Rose L, Lording D \& Liang S. Efficacy of tadalafil once daily in men with diabetes mellitus and erectile dysfunction. Diabetic Medicine 200825 138-146. (doi:10.1111/j.1464-5491.2007.02338.x)

41 Stirban A, Laude D, Elghozi JL, Sander D, Agelink MW, Hilz MJ \& Ziegler D. Acute effects of sildenafil on flow mediated dilatation and cardiovascular autonomic nerve function in type 2 diabetic patients. Diabetes/Metabolism Research and Reviews 200925 136-143. (doi:10.1002/dmrr.921)

42 Morano S, Mandosi E, Fallarino M, Gatti A, Tiberti C, Sensi M, Gandini L, Buchetti B, Lenti L, Jannini EA et al. Antioxidant treatment associated with sildenafil reduces monocyte activation and markers of endothelial damage in patients with diabetic erectile dysfunction: a double-blind, placebo-controlled study. European Urology $2007 \mathbf{5 2}$ 1768-1774. (doi:10.1016/j.eururo.2007.04.042)

43 Lee JW, Park HJ \& Park NC. Serum high-sensitivity C-reactive protein levels and response to $5 \mathrm{mg}$ tadalafil once daily in patients with erectile dysfunction and diabetes. Korean Journal of Urology $2013 \mathbf{5 4} 858-864$. (doi:10.4111/kju.2013.54.12.858)

44 La Vignera S, Condorelli RA, Vicari E, Lotti F, Favilla V, Morgia G, Maggi M \& Calogero AE. Seminal vesicles and diabetic neuropathy: ultrasound evaluation after prolonged treatment with a selective phosphodiesterase-5 inhibitor. Andrology $20131245-250$. (doi:10.1111/j.2047-2927.2012.00025.x)

45 Aversa A, Greco E, Bruzziches R, Pili M, Rosano G \& Spera G. Relationship between chronic tadalafil administration and improvement of endothelial function in men with erectile dysfunction: a pilot study. International Journal of Impotence Research 200719 200-207. (doi:10.1038/sj.ijir.3901513)

46 Rosano GM, Aversa A, Vitale C, Fabbri A, Fini M \& Spera G. Chronic treatment with tadalafil improves endothelial function in men with increased cardiovascular risk. European Urology $200547214-220$ (discussion 220-222). (doi:10.1016/j.eururo.2004.10.002)

47 Porst H, Brock GB, Kula K, Moncada I, Montorsi F, Basson BR, Kinchen K \& Aversa A. Effects of once-daily tadalafil on treatment satisfaction, psychosocial outcomes, spontaneous erections, and measures of endothelial function in men with erectile dysfunction but naive to phosphodiesterase type 5 inhibitors. Journal of Andrology 2012 33 1305-1322. (doi:10.2164/jandrol.111.015289)

48 Palmerini CA, Zucchi A, Fioretti F \& Arienti G. Erectile dysfunction and NO: variations of NO penile blood levels before and after sildenafil treatment. International Journal of Impotence Research 200921 321-325. (doi:10.1038/ijir.2009.33)

49 Alberti KG \& Zimmet PZ. Definition, diagnosis and classification of diabetes mellitus and its complications. Part 1: diagnosis and classification of diabetes mellitus provisional report of a WHO consultation. Diabetic Medicine 199815 539-553. (doi:10.1002/ (SICI)1096-9136(199807)15:7 < 539::AID-DIA668 > 3.0.CO;2-S)

50 Dishy V, Sofowora G, Harris PA, Kandcer M, Zhan F, Wood AJ \& Stein CM. The effect of sildenafil on nitric oxide-mediated vasodilation in healthy men. Clinical Pharmacology and Therapeutics 200170 270-279. (doi:10.1067/mcp.2001.117995)

51 Katz SD, Balidemaj K, Homma S, Wu H, Wang J \& Maybaum S. Acute type 5 phosphodiesterase inhibition with sildenafil enhances flowmediated vasodilation in patients with chronic heart failure. Journal of the American College of Cardiology 200036 845-851. (doi:10.1016/ S0735-1097(00)00790-7)

52 Kimura M, Higashi Y, Hara K, Noma K, Sasaki S, Nakagawa K, Goto C, Oshima T, Yoshizumi M \& Chayama K. PDE5 inhibitor sildenafil citrate augments endothelium-dependent vasodilation in smokers. Hypertension 200341 1106-1110. (doi:10.1161/01.HYP.0000068202.42431.CC) 
53 Vlachopoulos C, Tsekoura D, Alexopoulos N, Panagiotakos D, Aznaouridis K \& Stefanadis C. Type 5 phosphodiesterase inhibition by sildenafil abrogates acute smoking-induced endothelial dysfunction. American Journal of Hypertension 200417 1040-1044. (doi:10.1016/ j.amjhyper.2004.06.027)

54 Giannetta E, Feola T, Gianfrilli D, Pofi R, Dall'Armi V, Badagliacca R, Barbagallo F, Lenzi A \& Isidori AM. Is chronic inhibition of phosphodiesterase type 5 cardioprotective and safe? A meta-analysis of randomized controlled trials. BMC Medicine 201412 185-203. (doi:10.1186/s12916-014-0185-3)

55 Kaur J. A comprehensive review on metabolic syndrome. Cardiology Research and Practice 20142014 943162. (doi:10.1155/2014/943162)

56 Kojima H, Inoue T, Kunimoto H \& Nakajima K. IL-6-STAT3 signaling and premature senescence. JAK-STAT 20132 e25763. (doi:10.4161/ jkst.25763)

57 Lee IT \& Yang CM. Inflammatory signalings involved in airway and pulmonary diseases. Mediators of Inflammation 20132013791231. (doi:10.1155/2013/791231)

58 Badimon L, Romero JC, Cubedo J \& Borrell-Pages M. Circulating biomarkers. Thrombosis Research 2012130 (Suppl 1) S12-S15. (doi:10.1016/j.thromres.2012.08.262)
59 Messner B \& Bernhard D. Smoking and cardiovascular disease: mechanisms of endothelial dysfunction and early atherogenesis. Arteriosclerosis, Thrombosis, and Vascular Biology 201434 509-515. (doi:10.1161/ATVBAHA.113.300156)

60 Ross R. The pathogenesis of atherosclerosis: a perspective for the 1990s. Nature 1993362 801-809. (doi:10.1038/362801a0)

61 Solomon H, Man JW \& Jackson G. Erectile dysfunction and the cardiovascular patient: endothelial dysfunction is the common denominator. Heart 200389 251-253. (doi:10.1136/heart.89.3.251)

62 Thompson IM, Tangen CM, Goodman PJ, Probstfield JL, Moinpour CM \& Coltman CA. Erectile dysfunction and subsequent cardiovascular disease. Journal of the American Medical Association 2005294 2996-3002. (doi:10.1001/jama.294.23.2996)

63 Napoli C \& Ignarro LJ. Nitric oxide and pathogenic mechanisms involved in the development of vascular diseases. Archives of Pharmacal Research 200932 1103-1108. (doi:10.1007/s12272009-1801-1)

64 Schwartz BG, Jackson G, Stecher VJ, Campoli-Richards DM \& Kloner RA. Phosphodiesterase type 5 inhibitors improve endothelial function and may benefit cardiovascular conditions. American Journal of Medicine 2013126 192-199. (doi:10.1016/ j.amjmed.2012.08.015)

Received 13 August 2014

Revised version received 22 September 2014

Accepted 2 October 2014 